\title{
Essais
}

Revue interdisciplinaire d'Humanités

3 | 2013

Narration et lien social

\section{Entretien avec Maurice Fréchuret}

\section{François Trahais}

\section{(2) OpenEdition}

Journals

Édition électronique

URL : https://journals.openedition.org/essais/9480

DOI : 10.4000/essais. 9480

ISSN : 2276-0970

\section{Éditeur}

École doctorale Montaigne Humanités

\section{Édition imprimée}

Date de publication : 15 mai 2013

Pagination : 183-188

ISBN : 978-2-9544269-0-7

ISSN : $2417-4211$

Référence électronique

François Trahais, «Entretien avec Maurice Fréchuret », Essais [En ligne], 3 | 2013, mis en ligne le 01 septembre 2021, consulté le 08 septembre 2021. URL : http://journals.openedition.org/essais/9480 ; DOI : https://doi.org/10.4000/essais.9480 


\title{
Entretien avec Maurice Fréchuret
}

\author{
François Trahais
}

\section{Penser et pratiquer l'Histoire de l'art}

Votre pratique de l'Histoire de l'art se caractérise par de multiples approches de la discipline. En effet, vous êtes à la fois historien, conservateur de musée et commissaire d'exposition. En fonction de ces différents statuts, quelle est votre conception de l'Histoire de l'art ?

Penser et pratiquer l'Histoire de l'art est une chose qui est possible pour des personnes qui, comme moi, sont conservateur de Musée. Ce métier permet de penser l'histoire de l'art à travers les expositions que l'on conçoit et de pratiquer l'Histoire de l'art d'une manière privilégiée. Nous ne travaillons pas à partir d'images, nous travaillons avec des œuvres d'art et la différence est considérable. Les images sont des reproductions sur papier, sur diapositive ou sur support numérique. L'œuvre d'art, quant à elle, est un objet. Une œuvre est matérielle, c'est le fruit d'une fabrication, elle a un parcours et dès lors qu'il s'agit d'une œuvre assez ancienne son histoire est longue et compliquée. Elle a pu passer de mains en mains, appartenir à différents collectionneurs privés pour enfin aboutir dans une collection publique. Les œuvres d'art sont des objets qui nécessitent beaucoup d'attention et le métier de conservateur le laisse entendre. L'une des premières missions de ce métier est de faire en sorte que l'objet qui vous est confié, l'œuvre d'art dont vous avez la responsabilité, soit transmis aux générations futures dans le meilleur état possible pour que celles-ci puissent, à leurs tours, en bénéficier, pour pouvoir la contempler, l'admirer et l'étudier. Différentes méthodes permettent de veiller à la bonne conservation des œuvres comme la restauration préventive. Dans cette optique-là, il faut, par exemple, veiller à ne pas trop exposer à la lumière certaines œuvres pour qu'elles puissent conserver leurs couleurs initiales. La restauration curative quant à elle, doit résoudre des problèmes relevant d'un accident ou d'un acte de vandalisme. Mon métier me permet de dire les objets d'art ont un poids, une matière, une texture. Voilà pourquoi je souligne le fait que l'histoire de l'art telle que je la pense et telle que je la pratique repose sur des objets matériels et pas sur images. 


\section{Et le commissaire d'exposition procède d'une autre manière que celle du conservateur?}

En effet, l'exposition est une autre manière de pratiquer l'histoire de l'art. Il ne s'agit pas simplement d'accumuler des tableaux ou des sculptures, dans ce cas l'exposition serait mauvaise même si des chefs d'œuvres la composent. Une exposition c'est avant tout une réflexion qui part des œuvres. Le métier d'historien de l'art ou de conservateur doit toujours reposer sur la connaissance et la pratique des objets d'art. Une exposition c'est une histoire de l'art que les œuvres nous amènent à concevoir, à écrire et à montrer. Depuis maintenant quelques années, ma pratique repose sur plusieurs axes et s'il me faut dresser une typologie de mes pratiques de commissaire, je dois dire qu'après avoir organisé une quarantaine d'exposition, quatre catégories structurent mon approche du métier. En premier lieu, l'exposition la plus classique est l'exposition historique. Les œuvres d'art y sont liées par une période comme ce fut le cas dans les expositions 1946 ou encore Les Années $1970^{2}$. J'ai aussi mené des expositions thématiques à développement historique comme l'Envolée, l'enfouissement ${ }^{3}$. Notons également des expositions thématiques à développement anthropologique comme ce triptyque composé d'Un siècle d'arpenteur, les figures de la marche $4^{4}$, Dormir, rêver... et autres nuits et Hors d'auvre, ordre et désordres de la nourriture ${ }^{6}$. Enfin la quatrième catégorie concerne les expositions monographiques d'artistes.

\section{L'interdisciplinarité : 1'Histoire de l'art et la Sociologie}

\section{L'Histoire de l'art et la Sociologie, leur complémentarité et leurs inter- actions, semblent avoir guidé vos recherches. Quand et comment s'est manifesté l'intérêt que vous portez à ces deux disciplines ?}

J'ai bénéficié d'une double formation. Après 1968, j’avais alors vingt ans, année évidement importante dans la vie d'un jeune homme. J'ai commencé par faire des études de sociologie qui m’ont conduit à présenter quelques années plus tard un doctorat de $3^{\mathrm{e}}$ cycle. Je qualifierais ma formation de socio-

1 1946, l'art de la reconstruction. Musée Picasso, Antibes, juillet-août 1996.

2 Les années 1970, l'art en cause. CAPC Musée d'art contemporain de Bordeaux, 18 octobre 2002 19 janvier 2003.

3 L'envolée, l'enfouissement: Histoire et imaginaire aux temps précaires du XX'e siècle. Musée Picasso, Antibes, août-septembre 1995.

4 Les Figures de la marche, un siècle d'arpenteurs de Rodin à Neuman. Musée Picasso, Antibes,

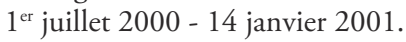

5 Dormir, rêver... et autres nuits. CAPC Musée d'art contemporain de Bordeaux, 3 février 21 mai 2006.

6 Hors d'auvre: ordre et désordres de la nourriture. CAPC Musée d'art contemporain de Bordeaux, 9 octobre 2004 - 13 février 2005. 
logue comme un peu vagabonde puisque mes professeurs étaient Pierre Sansot et Michel Maffesoli. Il y avait d'autres personnes comme Gilbert Durant, que je n'ai pas eu directement comme enseignants, mais dont les écrits m’ont beaucoup intéressé. Cette pensée s'opposait fortement à la sociologie officielle pratiquée notamment par Pierre Bourdieu. La sociologie à laquelle j'ai été formé était plus poétique, tournée vers l'imaginaire et je dois dire que cela a orienté ma vision de l'histoire de l'art. Une fois cette thèse présentée, je me suis rendu compte que ce n'était pas tout à fait ma voie. Après avoir enseigné les sciences sociales dans des écoles d'infirmières et dans des centres de formations pour travailleurs sociaux, je suis revenu à mes amours premières : l'Histoire de l'art. Ce n'était donc pas ma formation propre parce que je ne pouvais pas suivre les deux cursus en même temps. Cependant, dès l'âge de seize ou dix-sept ans, je m'intéressais déjà à l'art, aux ouvres d'art et particulièrement pour celles de mon époque, à la culture de mon temps. Je fis alors le constat suivant : je suis un homme du $\mathrm{XX}^{\mathrm{e}}$ siècle, j'utilise les outils du siècle, je suis complètement encré dans ce siècle et dans cette société, même si j'avais à son égard un regard très critique. Je ne peux pas imaginer que ma culture soit en décalage par rapport à cette période. Je me suis donc naturellement intéressé à l'art du $\mathrm{XX}^{\mathrm{e}}$ siècle. Le regard que je portais sur la société et sur son fonctionnement a trouvé dans l'histoire de l'art des références critiques, c'est pourquoi je me suis intéressé très tôt à Dada, au Surréalisme ainsi qu'aux groupes et aux mouvements qui s'opposaient aux dogmes et aux principes qui régissaient le fonctionnement culturel de la société.

\section{Comment s'est alors organisée votre formation d'Historien de l'art ?}

Je me souviens que dès l'âge de seize ou dix-sept ans j'ai écrit à Ben et à Arman pour vous dire à quel point l'art contemporain attirait déjà mon attention mais j'ai également compris très tôt qu'il ne fallait pas en rester là c'est pourquoi je suis remonté dans le passé et je me suis intéressé à toutes les formes d'art, qu'elles soient préhistoriques ou plus récentes. J'ai une grande passion pour l'art pariétal, je crois avoir visité en France toutes les grottes accessibles pour voir les dessins des artistes préhistoriques, $j$ 'ai même eu la très grande chance, il y a quelques années, de voir, avant qu'elle ne ferme définitivement, la grotte de Lascaux, l'originale, et je dois reconnaître que ce fut une émotion très forte. J'ai donc repris des études avec une équivalence au niveau de la maîtrise, j’ai ensuite passé un DEA en Histoire de l'art. Dix ans après mes études de sociologie, j’ai soutenu une thèse en Histoire de l'art au sein de l'Institut d'Histoire de l'art de Lyon. Cette thèse était consacrée à un artiste du XIX ${ }^{\mathrm{e}}$ siècle dont l'œuvre s'inscrit entre le symbolisme et le réalisme. Il s'agissait d'un travail sur Charles Maurin, natif de la région Rhône-Alpes, et qui correspondait aux critères de choix de mes professeurs de l'époque. J'avoue que mon choix aurait été différent si j'en avais eu la possibilité. J'aurai préféré consacrer ma thèse à l'étude d'une collection comme celle du Musée de Saint-Étienne mais les principes de l'Institut de l'Histoire de l'art à Lyon m'ont détourné de ce projet. J'ai cependant mené ces 
premières recherches sans déplaisir et même avec un certain enthousiasme parce que Charles Maurin était à mes yeux quelqu'un d'assez important. Il a enseigné la gravure à Felix Vallotton et à Toulouse-Lautrec, il fréquentait les milieux libertaires et ses écrits attestent de l'intérêt qu'il portait aux thèses anarchistes de l'époque. Tout ceci m'intéressait et m'amusait beaucoup. Une fois diplômé en Histoire de l'art, j'ai obtenu un poste de conservateur adjoint au sein du Musée de Saint-Étienne auprès de Bernard Ceysson, avec qui j’ai travaillé pendant près de quinze ans. Jacques Beauffet, qui était un ami d'adolescence a été l'un de mes principaux collaborateur pendant cette période.

À partir de 1979, ces quinze années furent extrêmement riches et fécondes dans la mesure où, Bernard Ceysson comme Jacques Beauffet, ont formé mon regard et initié mes pratiques de conservateur, de commissaire d'exposition et d'Historien de l'art.

Les années 1970 sont primordiales dans vos travaux d'historien et de commissaire au point d'y avoir consacré une exposition au sein du CAPC de Bordeaux il y a dix ans.

Je crois que c'est une décennie qui émerge plus tôt à tous les niveaux. Dès la fin des années 1960, une période formidable a débuté pour la création artistique, la littérature et les sciences humaines avec Michel Foucault et Gilles Deleuze par exemple. C'est un foisonnement extraordinaire de découvertes et de recherches. Cela me paraissait formidable, le jeune homme que j'étais à ce moment-là, ouvert à la culture contemporaine, ne pouvait que se réjouir de cette considérable propension des artistes à innover et à aboutir à des expérimentations des plus enthousiasmantes. Ces années 1960-1970 ont été déterminantes. Je suis convaincu qu'il y a deux très grands moments dans le $\mathrm{XX}^{\mathrm{e}}$ siècle : les années 1910 évidement avec l'émergence du cubisme, du cubofuturisme et de Dada ; et puis les années 1960-1970 qui remettent en cause tellement de choses, de manière tellement juste et passionnante. L'Histoire du CAPC de Bordeaux est liée à ce contexte, il s'inscrit dans cette effervescence artistique. En 2002, près de trente ans après l'ouverture du Musée, alors que je travaillais dans cette institution, le temps écoulé paraissait suffisant pour effectuer une mise au point sur l'art des années 1970 et c'est avec beaucoup de passion que j'ai pu échafauder les plans de cette exposition.

\section{III. Écrire l'Histoire de l'art}

\section{Deux formes de discours apparaissent dans votre pratique : l'exposition et la rédaction d'ouvrage. Quelle est la logique ou l'élément fédérateur de votre méthode critique?}

Le métier d'historien et celui de conservateur trouvent à s'enrichir mutuellement car les deux rôles se superposent et se complètent. Quand je suis conservateur de musée je me sens historien de l'art et lorsque je suis historien de l'art 
je reste malgré tout conservateur. Autrement dit, ce qui fait le lien ce sont les œuvres. Comme je l'ai déjà dit, elles sont bien plus que des images et la pratique de l'histoire de l'art en est changée. Cette pratique ne se limite pas à l'observation des ouvres, leur manipulation s'impose aussi lors des accrochages. Qu'il s'agisse d'un tableau, d'un dessin, d'une sculpture, d'une installation ou d'une vidéo, une certaine matérialité entre en jeu et cela est invisible dans un catalogue ou dans un livre. Ceci est très important : l'œuvre est centrale et ce sont toutes les œuvres que je vois ou que je manipule dans les musées, les expositions, les galeries et les centres d'art, qui m'enrichissent mon regard et ce sont elles qui sont mon point de départ. Elles sont le fil conducteur qui me permettra de d'organiser une exposition, d'écrire un texte, ou les deux en même temps. Il m'est arrivé de partir d'un projet d'exposition pour finalement aboutir à l'écriture d'un livre. Mon premier ouvrage publié en 1993 et qui s'intitule Le mou et ses formes ${ }^{7}$ était initialement conçu comme une exposition avec une typologie, un parcours et une démarche programmant l'élaboration d'une exposition. Pour des raisons diverses et variées celle-ci n'a pas eu lieu mais comme j'étais convaincu de l'intérêt du sujet, j'en ai fait un essai. J'ai parfois plus de chance, je pense une exposition en partant d'une sélection d'œuvre d'art, le projet aboutit et cela s'accompagne de la publication d'un livre ou d'un catalogue. Voilà comment les choses viennent à s'établir, je crois que ma méthode a pour principe premier de mettre l'œuvre d'art au cœur du dispositif de l'exposition et de l'écriture. Si l'on commence par une idée et que les œuvres d'art ne sont que des moyens d'illustrer cette idée, le projet échoue puisque les œuvres d'art deviennent de simples prétextes. C'est l'inverse qu'il faut pratiquer en se confrontant aux œuvres, en effectuant des rapprochements pour aboutir à une réflexion ou à un projet d'exposition.

\section{Vous avez choisi d'écrire une histoire de la sculpture au $\mathrm{XX}^{\mathrm{e}}$ siècle ${ }^{8}$ sous la forme d'un essai. En quoi ce genre littéraire sert-il votre pratique de l'Histoire de l'art?}

Un essai est une proposition, on s'essaye à quelque chose, c'est une réflexion. C'est un peu par modestie que l'on choisit le terme "essai ", pour éviter le caractère péremptoire d'un texte qui se voudrait définitif. Que ce soit dans Le mou et ses formes ${ }^{9}$ ou dans La machine à peindre ${ }^{10}$ c'est effectivement le mot essai que j'ai choisi parce que cela me paraissait être la forme la plus apte à mettre en évidence les catégories que j'avais envie d'explorer. Les années 1960-1970 sont celles d'artistes d'une génération légèrement supérieure à la mienne avec

7 Maurice Fréchuret, Le mou et ses formes, essai sur quelques catégories de la sculpture du XX'e siècle, éditions de l'École Nationale Supérieure des Beaux-Arts, Paris, 1993, 248 p.

8 Idem.

9 Ibid.

10 Maurice Fréchuret, La machine à peindre, éditions Jacqueline Chambon, Rayon Art, collection dirigée par Yves Michaud, Nîmes, 1994, 240 p. 
Claude Viallat en France, Robert Morris aux États-Unis, Mary Flanagan en Angleterre ou encore Giovanni Anselmo en Italie. Ces artistes produisaient au même moment des œuvres relevant des mêmes problématiques et des mêmes conditions matérielles sans qu'ils se soient réellement côtoyés. Ils engageaient, chacun dans leur coin, des démarches voisines et ressemblantes. Dans Le mou et ses formes, mon approche critique s'est appuyée sur des critères très physiques, très matériels, et déterminée selon ces trois catégories : «entasser, laisser pendre et nouer ». Les nœuds de Claude Viallat et ceux de Giovanni Anselmo ont effectivement quelque chose de très proche. Certes il faut souligner les différences de tonalités et de pratiques dans chez ces deux artistes, mais ils tous les deux questionner le geste de "nouer ». Pareil pour les tas : un grand nombre d'artistes de la même période ont entassé des choses. Qu'il s'agisse des land-artistes et de leur tas monumentaux, des petits tas de feuilles de Giuseppe Penone ou des tas de gravier de Bernard Pagès, la ressemblance de ces démarches devait être mis en évidence. "Faire tomber " et " laisser pendre » se sont aussi imposé comme de nouvelles pratiques chez de nombreux artistes de nationalités différentes et de manière simultanée. Voilà des catégories qui autorisent une autre manière de penser l'art et je suis assez opposé à une tradition de l'histoire de l'art qui consiste à étudier les mouvements les uns après des autres et sans faire de lien entre l'Arte Povera et Supports/Surfaces par exemple. D'abord parce que ces groupes n'ont jamais vraiment existé et je pense que l'exemple de Supports/ Surfaces est probant : il n'y a jamais eu d'exposition avec tous les artistes que l'on regroupe en général dans ce mouvement. Il y a eu beaucoup d'expositions historiques de Supports/Surfaces mais elles ont été tardives et l'unité du groupe est une construction artificielle a postériori. À l'exception de BMPT dont les protagonistes savaient que leur association n'allait pas durer, les groupes qui ont soi-disant émergé dans les années 1960-1970, étaient composés d'individus étonnements différents les uns des autres. Ils produisent des œuvres elles aussi extrêmement diverses et variées. Ces organisations étaient factices et artificielles, généralement nommées et orchestrées par des critiques d'art comme ce fut le cas avec Les Nouveaux Réaliste de Pierre Restany, d'où l'intérêt de penser et de pratiquer l'Histoire de l'art selon des catégories relevant des pratiques artistiques.

\section{Entretien mené par François Trahais,} doctorant en Histoire de l'art.

EA 538 Centre François-Georges Pariset Université Michel de Montaigne Bordeaux 3 francois.trahais@u-bordeaux3.fr

NDLR : Entretien réalisé le mardi 29 janvier 2013 dans le cadre des "Horizons de la Recherche » de l'École Doctorale Montaigne-Humanités. Penser et pratiquer l'Histoire de l'art par Maurice Fréchuret, Directeur des Musées Nationaux du XXe siècle des Alpes Maritimes. 